\title{
Programa Nacional de Melhoria do Acesso e da Qualidade da Atenção Básica (PMAQ-AB): modelização da política no âmbito nacional
}

\author{
Larissa Morgan Andrade Lemos \\ Mestre pela Universidade Federal da Bahia, \\ Vitória da Conquista, Bahia, Brasil \\ larissamorgan@hotmail.com \\ Nília Maria de Brito Lima Prado \\ Professora doutora da Universidade Federal da \\ Bahia, Vitória da Conquista, Bahia, Brasil \\ nilia.prado@ufba.br \\ Maria Guadalupe Medina \\ Professora doutora da Universidade Federal \\ da Bahia, Salvador, Bahia, Brasil \\ medina@ufba.br
}

Resumo Pretendeu-se identificar os elementos constituintes do arcabouço teórico do PMAQ-AB e, assim, sistematizar o modelo lógico deste programa. Para tanto, foi realizado estudo de avaliação que abrange revisão e análise de documentos, dentre os quais foram incluídos 35 referentes à intervenção proposta no âmbito nacional. Com base na elaboração do modelo foi possível compreender a plausibilidade lógica da proposta. Podese presumir que esse resultado permitirá a ampliação da visão de avaliação para as questões de organização relacionadas ao programa, ao tornar possível uma comparação entre o programa como fora previsto e o que tem sido, de fato, implementado nos diversos contextos dos municípios brasileiros.

Palavras-chave: Atenção primária à saúde, política de saúde, avaliação em saúde, avaliação de desempenho, avaliação da qualidade dos cuidados de saúde. 


\section{Introdução}

Nas últimas décadas, a Atenção Básica (AB) no Brasil ganhou destaque, especialmente desde a criação do Programa de Saúde da Família (PSF), quando houve significante expansão da cobertura, aumento considerável do acesso aos seus serviços e desenvolvimento de novos modelos de gestão e atenção (Teixeira et al., 2014). No entanto, ainda que essa expansão tenha sido acompanhada por diversas iniciativas de avaliação e de processos de indução da institucionalização da avaliação da Atenção Básica, é um desafio que permanece para gestores, profissionais e usuários, com vistas a assegurar a qualidade da atenção à saúde (Aleluia; Almeida; Medina, 2015).

Entre as iniciativas voltadas ao fortalecimento da avaliação no âmbito da Atenção Básica, destaca-se o Programa Nacional de Melhoria do Acesso e da Qualidade da Atenção Básica (PMAQ-AB) que, em 2011, por meio da Portaria n. 1.654, de 19 de julho, foi instituído pelo Ministério da Saúde (MS) como estratégia prioritária para alcançar mudanças de estrutura e organização de Unidades Básicas de Saúde (UBS) (Brasil, 2011a). O PMAQ-AB propõe-se a qualificar as práticas de gestão, cuidado e participação social e ampliar o acesso na Atenção Básica (Cruz et al., 2014; Fausto et al., 2014). Cabe ressaltar que o acesso diz respeito à universalidade, um dos princípios do SUS, e a qualidade se refere à integralidade da atenção à saúde, ante o cuidado incompleto.

A inovação creditada ao PMAQ-AB se dá por consistir em estratégia indutora da produção de uma cultura de análise, avaliação e intervenção que associa o incentivo financeiro ao desempenho de profissionais, à perspectiva de ampliação do financiamento e ao estabelecimento de indicadores e padrões de modo a tornar possível comparações estatísticas entre os resultados alcançados por diferentes gestões e equipes de saúde locais no âmbito da $\mathrm{AB}$, assim como pelas mesmas equipes e gestões em momentos distintos (Pinto; Sousa; Ferla, 2014; Mota; David, 2015; Rocha et al., 2016).

Por se tratar de um programa recente, as evidências científicas ainda são incipientes e restritas aos resultados quantitativos obtidos nos diferentes ciclos (Lopes, 2013; Machado et al., 2015; Pinto; Sousa; Florêncio, 2012; Linhares; Lira; Albuquerque, 2014; Rodrigues; Santos; Pereira, 2014;Santos et al.,2014;Barbosa, 2014;Lorena Sobrinho et al.,2015;Tomasi et al., 2015; Neves; Montenegro; Bittencourt, 2014). Na maioria dos estudos divulgados, não estão enfatizados os aspectos relacionados à análise de coerência e plausibilidade ou à racionalidade interna de funcionamento do programa.

Algumas evidências (Rossi; Lipsey; Freeman, 2004) destacam a importância de se partir da análise da teoria do programa para a identificação de fragilidades do desenho que poderão interferir no seu desempenho. Aferir a qualidade da teoria significa, em síntese, verificar se o programa está bem desenhado e se apresenta um plano plausível para o alcance dos resultados esperados. Ante o exposto, o propósito deste estudo foi identificar e delinear o modelo teórico lógico do PMAQ-AB no âmbito nacional. 


\section{Aspectos metodológicos}

Foi realizado um estudo de avaliação a respeito dos aspectos teóricos e lógicos do Programa Nacional de Melhoria do Acesso e da Qualidade da Atenção Básica no âmbito nacional. A elaboração do modelo lógico de uma intervenção consiste na determinação de seus componentes e de seu modo de operação, descrevendo todos os passos necessários para alcançar os seus propósitos e metas (Champagne et al., 2011).

Os dados foram produzidos com base na revisão da literatura normativa, técnica e científica, que permitiu identificar o conteúdo do programa em portarias reguladoras, manuais instrutivos, artigos científicos e dissertações.

A busca de informações foi realizada em bases de dados científicas e sites oficiais, no período de dezembro de 2017 a janeiro de 2018. Foram acessadas as bases de dados científicas da Biblioteca Virtual em Saúde (BVS) e da Scientific Electronic Library (Scielo); os sites oficiais do Sistema de Legislação da Saúde - Legis Saúde e do Portal da Saúde do Departamento de Atenção Básica do Ministério da Saúde - DAB/MS; e repositórios de Instituições de Ensino Superior (Quadro 1). Os descritores ou unitermos foram consultados na base de Descritores em Ciências da Saúde (DeCS) e, quando não identificados, empregou-se sinônimos e/ou palavras-chave utilizados para a edição de artigos científicos com a temática. As combinações dos unitermos e as estratégias de busca foram adequadas para cada base consultada.

Adicionalmente, realizou-se a busca de documentos normativos no site Legis Saúde, por meio do unitermo "Programa Nacional de Melhoria do Acesso e da Qualidade"; e os filtros de busca considerados foram: data da publicação, o tipo de norma (todas), fonte (todas) e situação (vigentes e revogadas). No Portal da Saúde do Departamento de Atenção Básica do Ministério da Saúde (DAB/MS), as legislações foram identificadas no campo Biblioteca > Legislações> PMAQ-AB, enquanto as demais publicações (manuais instrutivos e instrumentos para autoavaliação) foram extraídas no campo Biblioteca> Publicações> Cartilhas, Guias e Manuais. As dissertações foram identificadas nos repositórios institucionais por meio dos unitermos "Programa Nacional de Melhoria do Acesso e da Qualidade" e "PMAQ-AB". 
Quadro 1 - Tipificação dos documentos quanto às fontes de dados

\begin{tabular}{|c|c|c|c|}
\hline Tipologia & $\begin{array}{l}\text { Fonte de } \\
\text { dados }\end{array}$ & Documentos & $\begin{array}{l}\text { Número de } \\
\text { documentos }\end{array}$ \\
\hline $\begin{array}{c}\text { Bases de dados } \\
\text { científicas }\end{array}$ & BVS & $\begin{array}{l}\text { Mota; David, 2015; } \\
\text { Moraes; Iguti, } 2013 .\end{array}$ & 2 artigos \\
\hline $\begin{array}{c}\text { Bases de dados } \\
\text { científicas }\end{array}$ & Scielo & $\begin{array}{l}\text { Pinto; Sousa; Ferla, 2014; } \\
\text { Coelho e Silva et al., 2017; } \\
\text { Rocha et al., 2016; Pinto; } \\
\text { Sousa; Florêncio, } 2012 .\end{array}$ & 4 artigos \\
\hline $\begin{array}{l}\text { Repositórios } \\
\text { institucionais }\end{array}$ & $\begin{array}{c}\text { UFRGS, } \\
\text { UNB, UFPE }\end{array}$ & $\begin{array}{c}\text { Pinto, 2014; Cavalcanti, } \\
\text { 2014; Gomes, } 2013 .\end{array}$ & 3 dissertações \\
\hline $\begin{array}{l}\text { Sistema de } \\
\text { Pesquisa de } \\
\text { Legislação }\end{array}$ & Saúde Legis & $\begin{array}{l}\text { Brasil, 2011a; Brasil, 2011e; Brasil, } \\
\text { 2012a; Brasil, 2012b; Brasil, 2012c; } \\
\text { Brasil, 2012d; Brasil, 2012e; } \\
\text { Brasil, 2012f; Brasil, 2013b; Brasil, } \\
\text { 2013c; Brasil, 2013d; Brasil, } \\
\text { 2013e; Brasil, 2013f; Brasil, 2014a; } \\
\text { Brasil, 2014b; Brasil, 2015a; } \\
\text { Brasil, 2015b; Brasil, 2016; } \\
\text { Brasil, 2016b. }\end{array}$ & 19 portarias \\
\hline Site oficial & $\begin{array}{l}\text { Portal do } \\
\text { Ministério } \\
\text { da Saúde }\end{array}$ & $\begin{array}{c}\text { Brasil, 2011b; Brasil, 2011c; } \\
\text { Brasil, 2011d; Brasil, 2012g; } \\
\text { Brasil, 2012h; Brasil, 2013a; } \\
\text { Brasil, 2013g; Brasil, } 2017 .\end{array}$ & $\begin{array}{l}5 \text { portarias } \\
\text { e } 3 \text { manuais } \\
\text { técnicos }\end{array}$ \\
\hline
\end{tabular}

Fonte: Elaborado pelos autores, 2018.

Foram identificados 47 documentos e, depois de leitura na íntegra, foram excluídos 4 artigos científicos e 8 portarias por tratarem de temática diferente do objeto de interesse. Ao final, foram incluídos 36 documentos divulgados entre os anos de 2011 a 2017, que descreviam e discutiam a formulação e a implementação do PMAQ-AB no âmbito nacional (Quadro 1).

Para análise de coerência, os excertos dos documentos foram submetidos a um processo de análise e sistematização das seguintes informações: identificação do problema que originou o programa (descritores, se possível) e proposições para formulação do programa (Figura 1), assim como, objetivo geral, objetivos específicos, população alvo, atividades previstas, resultados e estrutura lógica do programa (Figura 2).

Este artigo compõe o projeto de dissertação de mestrado intitulado Modelização do Programa Nacional de Melhoria do Acesso e da Qualidade em um município baiano, aprovado pelo Comitê de Ética em Saúde da Universidade Federal da Bahia do Instituto Multidisciplinar em Saúde, sob o parecer n. 2.475.022. 


\section{Resultados e discussão}

A análise de documentos permitiu a sistematização de três subtópicos que constituíram os resultados, quais sejam: reconhecimento da necessidade do programa, formulação da proposta e modelo teórico lógico do PMAQ-AB no Brasil.

\section{Reconhecimento da necessidade do programa}

Os principais problemas que fomentaram os debates acerca da necessidade de um programa voltado para a ampliação do acesso e da qualidade no âmbito da Atenção Básica foram: o subfinanciamento federal para o desenvolvimento das ações; a incipiência dos processos de gestão centrados na indução e acompanhamento da qualidade; a necessidade de qualificação do processo de trabalho (alta rotatividade profissional, sobrecarga de trabalho, incipiente integração das equipes de $\mathrm{AB}$ com os outros pontos da Rede de Atenção à Saúde, a persistência do modelo de queixa-conduta, procedimentos centrados no médico, foco na dimensão biomédica do processo saúde-doença-cuidado); e as condições de infraestrutura das unidades de assistência (precariedade das instalações fisicas, condições de trabalho) (Figura 1).

Figura 1 - Sistematização dos fatores motivadores para a proposição de um programa de melhoria do acesso e da qualidade na Atenção Básica

\begin{tabular}{|c|c|}
\hline DESAFIOS A SEREM ENFRENTADOS & SITUACYAO GERADA PELOS DESAFIOS \\
\hline Fiaanciameato iasuficieate e iasdequado da $\mathrm{AB}$. & Dificuldade na manuteaçà e expausào dos serviços. \\
\hline $\begin{array}{l}\text { Incipiencia dos processos de gestào centrados na indaçào e } \\
\text { acompanlamento da qualidade. }\end{array}$ & Coberfura e qualidade das açōes comprometida. \\
\hline \multirow{4}{*}{ 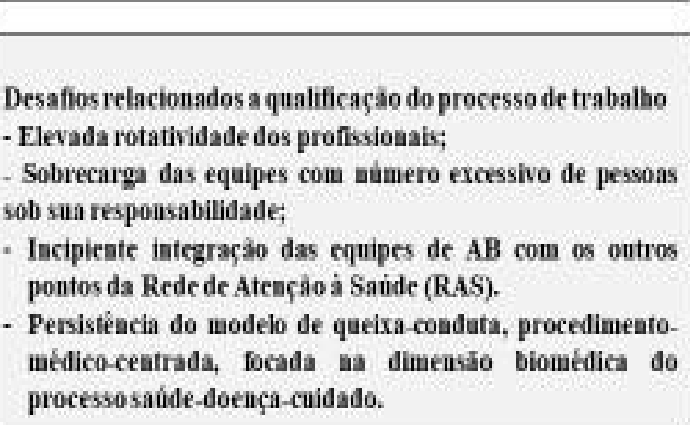 } & $\begin{array}{l}\text { Comprometimento da integralidade de assistencia e baixa } \\
\text { resolutividade }\end{array}$ \\
\hline & $\begin{array}{l}\text { Desconiecimento do desempenho das equipes e dos resultados } \\
\text { das práticas desenvolvidas frente os indicadores de saude }\end{array}$ \\
\hline & $\begin{array}{l}\text { Incipieate orieatação đo trabalho em função de prioridades, } \\
\text { metase resultados. }\end{array}$ \\
\hline & $\begin{array}{l}\text { Dificuldades de iutegraçào da equipe e pouca } \\
\text { formaça de vinculos, o que compromete a continuudade do } \\
\text { cuidado. }\end{array}$ \\
\hline \multirow{2}{*}{$\begin{array}{l}\text { Desafios relacionados as condiçōes de infraestrutura đas } \\
\text { unidades assistenciais: } \\
\text { - Precariedade da rede fisica além da ambiència pouco } \\
\text { acolledora das UBS. } \\
\text { - Inadequadas condiçóes de traballıo para os profissionais }\end{array}$} & $\begin{array}{l}\text { Comprometimeato đa capacidade de iatervenção dos } \\
\text { profissionais e satisfação com o traballo. }\end{array}$ \\
\hline & $\begin{array}{l}\text { Faks percepçào de que os servicos ofertados sijo de baixa } \\
\text { qualidade. }\end{array}$ \\
\hline
\end{tabular}

Fonte: Elaborada pelos autores, 2018. 
A pouca sustentabilidade política e financeira proveniente de um financiamento insuficiente que assola a Atenção Básica, principalmente a partir da expansão da cobertura populacional da ESF, tem sido considerada por alguns autores como o ponto de partida que fomentou discussões sobre a necessidade da ampliação de recursos federais e de ações de melhoria do acesso e de qualificação das práticas (Cavalcanti, 2014; Rocha et al., 2016; Pinto, 2014).

Essa situação teve como consequências múltiplas análises de diferentes atores, especialmente quanto a estratégias que modificassem a composição de recursos do âmbito federal, sendo proposta a transferência de montantes per capita, repasses para projetos prédefinidos e para investimento em infraestrutura vinculados à implantação de programas prioritários e, por fim, recursos condicionados a resultados e avaliação do acesso e da qualidade. Este último mecanismo de repasse reforça a proposta governamental de formulação de um programa capaz de realizar avaliações contínuas e induzir mudanças de práticas.

A proposta em discussão inspirou-se em experiências internacionais com sistemas que já associavam a avaliação do desempenho e/ou resultados a um componente financeiro denominado pagamento por desempenho (pay for performance), ou financiamento baseado em resultados (Berenson; Rich, 2010; Pearson et al., 2008; Girardi; Salgado;Van Stralen, 2015). Países como Austrália, Canadá, EUA, Nova Zelândia, Portugal e Reino Unido, embora apresentem sistemas de saúde com características de organização distintas, fundamentam a remuneração por desempenho na lógica de que consequências positivas induzem comportamentos que refletem na melhoria de resultados (Sousa,2011;Alshamsan et al., 2010; Kondo et al., 2016). Contudo, duas questões sinalizavam o desafio para a consolidação dessa estratégia: a insuficiência dos sistemas de informação disponíveis na atenção primária, o que compromete as comparações entre os desempenhos das equipes; e as assimetrias regionais que não são consideradas no processo de contratualização dos indicadores e metas (Perelman et al., 2016; Sousa, 2011). Tais questões também foram apontadas em estudos nacionais e reforçam a premência de um programa que consiga contornar esses problemas.

A qualificação das práticas de atenção e da gestão com maior inclusão da avaliação e acompanhamento também tem sido assinalada como estratégia para dar legitimidade ao planejamento e a tomada de decisões, especialmente no âmbito local, com o intuito de evitar a proliferação de ações pontuais e fragmentadas e permitir a integralidade da atenção (Felisberto, 2006).

Do mesmo modo, os problemas relacionados às condições de infraestrutura enfrentados pela Atenção Básica brasileira são apontados em estudos comparados do Brasil com outros países. Souza et al. (2013) destacam que, além de espaços físicos inadequados e déficit de material, as equipes incompletas e fragmentação do cuidado também são fatores que têm comprometido o acesso e a qualidade da atenção.

Esse conjunto de fatores descortinam outras fragilidades que reforçam a necessidade de inclusão de iniciativas para fortalecer a atenção primária, quais sejam: obstáculos na manutenção e expansão dos serviços; desconhecimento do desempenho de equipes 
e de resultados das práticas desenvolvidas diante de indicadores de saúde; incipiente orientação do trabalho em razão de prioridades, metas e resultados; óbices de integração da equipe e pouca formação de vínculos com os usuários; cobertura e qualidade de ações comprometidas; baixa integralidade e resolutividade da assistência; comprometimento da capacidade de intervenção dos profissionais e satisfação com o trabalho; falsa percepção de que os serviços ofertados são de baixa qualidade.

\section{Formulação da proposta}

As discussões acerca dos desafios existentes no âmbito da atenção primária suscitaram a formulação da Portaria n. 1.654 em 19 de julho de 2011, que instituiu o Programa Nacional de Melhoria do Acesso e da Qualidade. Este tem por objeto principal:

Induzir a ampliação do acesso e a melhoria da qualidade da atenção básica, com garantia de um padrão de qualidade comparável nacional, regional e localmente, de maneira a permitir maior transparência e efetividade das ações governamentais direcionadas à Atenção Básica em Saúde. (Brasil, 2011a, p. 1; Brasil, 2012g, p. 7; Brasil, 2013g, p. 6; Brasil, 2017, p. 8).

Este objetivo se manteve inalterado em todos os outros documentos normativos publicados nos anos subsequentes e norteou a organização dos três ciclos do programa, ocorrendo o mesmo com os objetivos específicos, que de forma idêntica compuseram a estrutura dos três manuais instrutivos já divulgados. Entre estes cabe destacar: ampliar o impacto da $\mathrm{AB}$ sobre as condições de saúde da população e sobre a satisfação dos seus usuários; fornecer padrões de boas práticas e organização das UBS que norteiem a melhoria da qualidade; promover maior conformidade das UBS com os princípios da $\mathrm{AB}$; promover a qualidade e a inovação na gestão da $\mathrm{AB}$; melhorar a qualidade da informação e o uso de Sistemas de Informação como recurso de gestão da AB; instituir uma cultura de avaliação da $A B$ no SUS e de gestão com base na indução e acompanhamento de processos e resultados; e estimular o foco da $\mathrm{AB}$ no usuário, promovendo a transparência dos processos de gestão, a participação e controle social e a responsabilidade sanitária dos profissionais e gestores (Brasil, 2012a; Brasil, 2013g; Brasil, 2017).

Quanto às diretrizes do Programa e as fases de operação que o constituem, essas se encontravam implícitas em um conjunto de documentos normativos e técnicos (Brasil, 2011a; Brasil, 2012g; Brasil, 2013g; Brasil, 2017). Apesar da Portaria n. 1.654 ter sido publicada em 2011, não especificava de modo claro e detalhado as diretrizes orientadoras do processo de organização do programa pelos municípios que aderiram no primeiro ano (Brasil, 2011a).As diretrizes apresentadas em tal documento normativo foram as seguintes: elaborar referência de comparação entre as equipes considerando as diferentes realidades de saúde; estimular um processo contínuo e progressivo de melhorias dos padrões e indicadores de acesso e de qualidade por contínuo ajuste e novos pactos de indicadores e padrões; garantir mecanismos de transparência de todas as etapas; envolver, mobilizar e tornar responsáveis os gestores federal, estaduais, municipais e locais, equipes e usuários 
num processo de mudança de cultura de gestão; desenvolver cultura de negociação e contratualização que implique a gestão dos recursos relacionados a compromissos e resultados estabelecidos sob pactos e alcançados; estimular a efetiva mudança do modelo de atenção; ter caráter voluntário para a adesão.

Contudo, tais diretrizes, em razão do caráter amplo e abstrato, provocaram algumas contestações sobre quais estratégias deveriam ter prioridade para execução de ações, com o intuito de tornar possível a transparência das ações de governo, garantir a adesão voluntária, e também que comparações entre equipes estimulassem a melhoria contínua sem provocar a competição entre elas (Mota; David, 2015).

Somente em 2012, com a edição do primeiro manual instrutivo do PMAQ-AB, tais diretrizes foram reapresentadas de modo a vincular os objetivos específicos às ações necessárias para a execução do programa (Brasil, 2012g). O que indica que na fase inicial do programa os atores envolvidos na implementação podem ter apresentado dificuldades em compreender o que de fato estava sendo proposto.

Em relação ao exposto, alguns autores assinalaram que o PMAQ-AB, principalmente no seu primeiro ciclo, foi organizado pela execução de etapas consecutivas e estanques, de modo vertical, "desconsiderando o jogo de interesses, os vários atores implicados e as diferenças nos cenários de implementação da política” (Rocha et al., 2016. p. 25), como se apenas a norma pudesse garantir a implementação do programa. Cabe ressaltar que mesmo após a reformulação de documentos técnicos e normativos, tais características permaneceram frágeis quanto aos aspectos orientadores do processo de implementação.

As ações que sustentam os objetivos do programa são desenvolvidas de modo cíclico e, inicialmente, se observava quatro fases: (1) a adesão ao programa ocorre por contratos de compromissos e indicadores de avaliação, (2) o desenvolvimento do conjunto de ações que serão empreendidas pelas EAB e gestão das três esferas de governo, (3) a avaliação externa, que almeja certificar as equipes por meio de análise dos indicadores contratados e averiguação de um conjunto de padrões de qualidade e (4) a recontratualização, constituída por um processo de pacto de novos padrões e indicadores de qualidade, para estimular um processo cíclico e sistemático de melhoria com base em resultados alcançados pelos participantes do PMAQ-AB. Entretanto, com o lançamento da Portaria n. 1.645, de 2 de outubro de 2015, o desenvolvimento passou a ser Eixo Estratégico Transversal, reforçando o caráter cíclico e contínuo do programa (Cavalcanti; Oliveira Neto; Sousa, 2015).

Depois das edições das primeiras portarias que caracterizavam o PMAQ-AB, o Ministério da Saúde procurou organizar o processo de adesão dos municípios e das equipes de Atenção Básica ao primeiro ciclo do programa (Quadro 2).

A partir da adesão, uma parte do recurso financeiro instituído pelo PMAQ-AB é repassada para os municípios para que iniciem a organização das atividades vinculadas ao processo de melhoria, por meio da definição e instituição de padrões (Pinto; Sousa; Florêncio, 2012). Apesar da grande importância do componente financeiro no êxito dos propósitos do PMAQ-AB, a Portaria n.1.654/2011 regulou apenas que ele fosse realizado/ 
transferido fundo a fundo (do plano federal para o municipal), não sinalizando de que modo exatamente esses municípios deveriam aplicar esse recurso. Isso permite que os municípios estabeleçam normas específicas para a sua utilização (por meio de adicionais pecuniários e/ou despesas em suprimentos, infraestrutura e outros estímulos que possam auxiliar a melhoria do desempenho das equipes) (Moraes; Iguti, 2013). A indefinição quanto a aplicação de recurso proveniente da avaliação do PMAQ-AB permite que o município opte por investir naquelas equipes que obtiveram maior grau de desempenho ou, alternativamente, investir nas equipes que obtiveram as pontuações mais baixas, para promovê-las. Contudo esse leque de opções pode gerar consequências não intencionais e, consequentemente, contribuir para a ampliação das desigualdades locais (Macinko; Harris; Rocha, 2017).

Quanto ao componente de avaliação e monitoramento, o PMAQ-AB recomendou a autoavaliação e as avaliações externas. Para a autoavaliação, o programa instituiu um instrumento de avaliação, o AMAQ (Autoavaliação para Melhoria do Acesso e da Qualidade da Atenção Básica). Esse instrumento busca promover reflexões sobre as responsabilidades, estabelecer padrões de qualidade que tenham caráter de incremento e permitir a quantificação das respostas autoavaliativas (Brasil, 2012a). Entretanto, a autoavaliação não é obrigatória, apesar de sua aplicação refletir o momento de avaliação externa, que pode ser considerado, efetivamente, o ato avaliativo propriamente dito.

A avaliação externa é o momento posterior ao desenvolvimento de possíveis mudanças pleiteadas depois da adesão ao programa. Esse processo é apoiado por diferentes atores, gestores dos três níveis federados, algumas instituições de ensino superior e centros de pesquisa. Essa avaliação constituirá um escore de comparação, conforme normas estabelecidas por distintos documentos (Brasil, 2011a; Brasil, 2012c; Brasil, 2013c), que preconiza o acompanhamento quanto a realização da autoavaliação, o desempenho da $\mathrm{EAB}$ nos indicadores estabelecidos sob pactos e acompanhados durante toda a fase de desenvolvimento e o resultado da aplicação dos instrumentos de avaliação da terceira fase. $\mathrm{O}$ resultado está vinculado à determinação do percentual de recurso repassado mensalmente ao Fundo Municipal de Saúde pelo Ministério da Saúde (Brasil, 2012a; Brasil, 2012b; Brasil, 2013a; Brasil, 2017).

A recontratualização de compromissos, o acompanhamento de indicadores e o alcance de resultados faz parte de um modelo de avaliação de desempenho que procura mensurar os possíveis efeitos da Política Nacional de Atenção Básica com a finalidade de subsidiar os processos de tomada de decisão, dar clareza aos resultados alcançados, além de garantir a transparência dos processos de gestão do SUS e intensificar o controle social. 
Quadro 2 - Sistematização dos documentos normativos relacionados a implementação do PMAQ-AB e execução do primeiro ciclo do programa

\begin{tabular}{|c|c|c|c|}
\hline \multicolumn{4}{|c|}{ Documentos Normativos } \\
\hline Mês/Ano & Documento & Principal objetivo & Referência \\
\hline $07 / 2011$ & $\begin{array}{l}\text { Portaria } \\
\text { n. } 1.654\end{array}$ & $\begin{array}{l}\text { Instituir o PMAQ-AB, com a finalidade de induzir } \\
\text { a ampliação do acesso e a melhoria da qualidade da } \\
\text { atenção básica, garantindo um padrão de qualidade } \\
\text { comparável nacional, regional e localmente, de } \\
\text { maneira a permitir maior transparência e efetividade } \\
\text { das ações governamentais direcionadas à Atenção } \\
\text { Básica em Saúde. }\end{array}$ & $\begin{array}{l}\text { Brasil, } \\
\text { 2011a. }\end{array}$ \\
\hline 09/2011 & $\begin{array}{l}\text { Portaria } \\
\text { n. } 576\end{array}$ & $\begin{array}{c}\text { Estabelecer novas regras para a Carga Horária } \\
\text { Semanal (CHS) dos médicos, enfermeiros e } \\
\text { cirurgiões-dentistas. Estabelecer normas para o } \\
\text { cadastramento no SCNES (Sistema de Cadastro Nacional } \\
\text { de Estabelecimentos de Saúde) das Equipes de Atenção } \\
\text { Básica que farão parte do PMAQ-AB. Incluir na Tabela de } \\
\text { Tipo de Equipes do SCNES, os tipos de equipes conforme } \\
\text { os anexos desta portaria. }\end{array}$ & $\begin{array}{l}\text { Brasil, } \\
\text { 2011b. }\end{array}$ \\
\hline $10 / 2011$ & $\begin{array}{l}\text { Portaria } \\
\text { n. } 2.396\end{array}$ & $\begin{array}{l}\text { Definir o valor mensal integral do incentivo } \\
\text { financeiro do PMAQ-AB denominado como } \\
\text { Componente de Qualidade do Piso de Atenção } \\
\text { Básica Variável (PAB Variável). }\end{array}$ & $\begin{array}{l}\text { Brasil, } \\
\text { 2011c. }\end{array}$ \\
\hline $10 / 2011$ & $\begin{array}{l}\text { Portaria } \\
\text { n. } 2.488\end{array}$ & $\begin{array}{l}\text { Aprovar a Política Nacional de Atenção Básica, } \\
\text { com vistas à revisão da regulamentação de } \\
\text { implantação e operacionalização vigentes. }\end{array}$ & $\begin{array}{l}\text { Brasil, } \\
\text { 2011d. }\end{array}$ \\
\hline $11 / 2011$ & $\begin{array}{l}\text { Portaria } \\
\text { n. } 2.812\end{array}$ & $\begin{array}{l}\text { Homologar a adesão dos Municípios e das } \\
\text { respectivas equipes de saúde da Atenção Básica } \\
\text { ao Programa Nacional de Melhoria do Acesso e da } \\
\text { Qualidade da Atenção Básica (PMAQ-AB). }\end{array}$ & $\begin{array}{l}\text { Brasil, } \\
\text { 2011e. }\end{array}$ \\
\hline $02 / 2012$ & $\begin{array}{c}\text { Portaria } \\
\text { n. } 225\end{array}$ & $\begin{array}{l}\text { Homologar a adesão dos Municípios e das } \\
\text { respectivas equipes de saúde da Atenção Básica } \\
\text { ao Programa Nacional de Melhoria do Acesso e da } \\
\text { Qualidade da Atenção Básica (PMAQ-AB), } \\
\text { complementando a Portaria n. } 2812 / 2011 \text {. }\end{array}$ & $\begin{array}{l}\text { Brasil, } \\
\text { 2012a. }\end{array}$ \\
\hline
\end{tabular}




\begin{tabular}{|c|c|c|c|}
\hline \multicolumn{4}{|c|}{ Documentos Normativos } \\
\hline Mês/Ano & Documento & Principal objetivo & Referência \\
\hline $04 / 2012$ & $\begin{array}{c}\text { Portaria } \\
\text { n. } 644\end{array}$ & $\begin{array}{l}\text { Homologar a adesão dos Municípios e das } \\
\text { respectivas Equipes de Atenção Básica ao Programa } \\
\text { Nacional de Melhoria do Acesso e da Qualidade da } \\
\text { Atenção Básica (PMAQ-AB), complementando a } \\
\text { Portaria n. 2812/2011 e a Portaria n. 225/2012. }\end{array}$ & $\begin{array}{l}\text { Brasil, } \\
\text { 2012b. }\end{array}$ \\
\hline $05 / 2012$ & $\begin{array}{c}\text { Portaria } \\
\text { n. } 866\end{array}$ & $\begin{array}{l}\text { Alterar o prazo para solicitação da avaliação } \\
\text { externa no Programa Nacional de Melhoria do } \\
\text { Acesso e da Qualidade da Atenção Básica (PMAQ- } \\
\text { AB) e as regras de classificação da certificação das } \\
\text { equipes participantes do Programa. }\end{array}$ & $\begin{array}{l}\text { Brasil, } \\
\text { 2012c. }\end{array}$ \\
\hline $05 / 2012$ & $\begin{array}{l}\text { Portaria } \\
\text { n. } 1.089\end{array}$ & $\begin{array}{c}\text { Definir o valor mensal integral do incentivo } \\
\text { financeiro do PMAQ-AB, denominado como } \\
\text { Componente de Qualidade do Piso de Atenção } \\
\text { Básica Variável. }\end{array}$ & $\begin{array}{l}\text { Brasil, } \\
\text { 2012d. }\end{array}$ \\
\hline $11 / 2012$ & $\begin{array}{l}\text { Portaria } \\
\text { n. } 2.626\end{array}$ & $\begin{array}{l}\text { Autorizar o repasse do incentivo financeiro } \\
\text { do Programa Nacional de Melhoria do Acesso e } \\
\text { da Qualidade da Atenção Básica (PMAQ-AB), } \\
\text { denominado como Componente de Qualidade do } \\
\text { Piso de Atenção Básica Variável. }\end{array}$ & $\begin{array}{l}\text { Brasil, } \\
\text { 2012e. }\end{array}$ \\
\hline $12 / 2012$ & $\begin{array}{l}\text { Portaria } \\
\text { n. } 3.114\end{array}$ & $\begin{array}{l}\text { Autorizar o repasse do incentivo financeiro } \\
\text { do Programa Nacional de Melhoria do Acesso e } \\
\text { da Qualidade da Atenção Básica (PMAQ-AB), } \\
\text { denominado como Componente de Qualidade do } \\
\text { Piso de Atenção Básica Variável. }\end{array}$ & $\begin{array}{l}\text { Brasil, } \\
\text { 2012f. }\end{array}$ \\
\hline $03 / 2013$ & $\begin{array}{c}\text { Portaria } \\
\text { n. } 283\end{array}$ & $\begin{array}{l}\text { Autoriza o repasse do incentivo financeiro } \\
\text { do Programa Nacional de Melhoria do Acesso e } \\
\text { da Qualidade da Atenção Básica (PMAQ-AB), } \\
\text { denominado como Componente de Qualidade do } \\
\text { Piso de Atenção Básica Variável. }\end{array}$ & $\begin{array}{l}\text { Brasil, } \\
\text { 2013a. }\end{array}$ \\
\hline
\end{tabular}

Fonte: Elaborado pelos autores, 2018.

\section{Modelo Lógico}

O modelo lógico do PMAQ-AB foi elaborado com reconhecimento da situaçãoproblema vivida pela Atenção Básica no momento em que antecedeu a formulação da sua proposta. Esses transtornos propiciaram a identificação mais nítida da dimensão prioritária do programa, objetivos que os compõe, ações e resultados pretendidos pelo programa. Este modelo foi representado por meio de uma figura ilustrativa (Figura 2). 
No modelo consta como dimensão a organização das práticas avaliativas relacionadas aos seguintes objetivos específicos: viabilizar a comparação entre as equipes de saúde, instituir mecanismos de avaliação da $\mathrm{AB}$, estimular uma progressiva e contínua melhoria dos indicadores de acesso e qualidade, instituir mecanismos de financiamento da $\mathrm{AB}$ associados a avaliação do desempenho das equipes de saúde, estimular a qualificação dos processos de gestão e trabalho dos profissionais e a responsabilidade compartilhada entre todos os atores envolvidos no processo de implementação do programa, garantir a voluntariedade no processo de adesão das equipes e dos gestores de Atenção Básica municipal ao PMAQ-AB e viabilizar mecanismos que possibilitem a transparência e o acompanhamento das ações referentes ao PMAQ-AB pela sociedade.

Tais objetivos derivam nas ações relacionadas à pactuação de indicadores para avaliação do desempenho das equipes, estabelecimento de padrões para avaliação do acesso e qualidade pelas equipes pertencentes a um mesmo estrato, estabelecimento de valores a serem transferidos para as equipes de acordo com o desempenho alcançado, inclusão de estratégias para a formação permanente, inclusive quanto às práticas de avaliação em saúde, utilização dos resultados da avaliação para o planejamento de novas estratégias para ampliação do acesso e da qualidade e adequação dos processos de trabalho das equipes de saúde e da gestão local a cada ciclo do programa.

Com esses resultados, a curto e longo prazo poderá ser percebido o ponto focal para a resolução do problema que deu origem ao programa: equipes mais estimuladas quanto ao desenvolvimento das práticas; avaliação institucionalizada no processo de trabalho das equipes e gestão; ampliação dos recursos a serem aplicados na Atenção Básica, provenientes do Componente de Qualidade do Piso de Atenção Básica Variável proporcional ao desempenho verificado na certificação; processos de trabalho e de gestão qualificados de acordo com as diretrizes da PNAB; usuários envolvidos no programa, acompanhando o desempenho das equipes e da gestão municipal através dos Conselhos Locais e Municipais de Saúde; profissionais e gestores utilizando os resultados das avaliações nas práticas de planejamento das ações; e, consequentemente, a ampliação do acesso e da melhoria da qualidade da atenção básica. 
Figura 2 - Modelo lógico do Programa Nacional de Melhoria do Acesso e da Qualidade da Atenção Básica
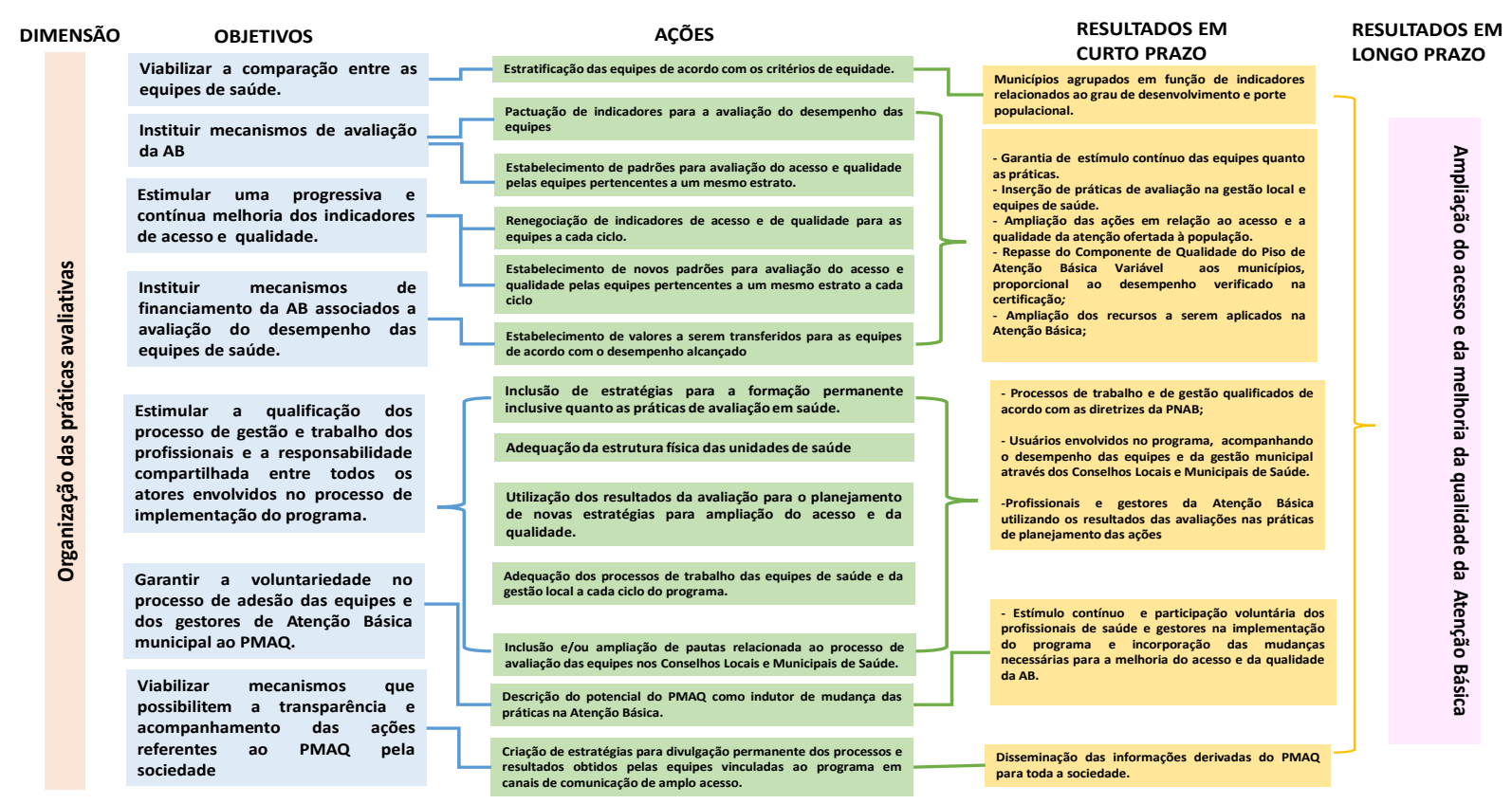

Fonte: Elaborada pelos autores, 2018.

\section{Considerações finais}

O PMAQ-AB é um programa de grande envergadura que foi instituído em um curto período de tempo. Por isso, existem poucos estudos sobre o desenho do PMAQ$\mathrm{AB}$ no âmbito nacional que tratem do contexto e do processo político que influencia o desenvolvimento do programa.

Este artigo procurou descrever a principal dimensão, os objetivos pretendidos, bem como as atividades e os principais resultados a curto e longo prazo, além de elaborar, com base nesses elementos, o modelo lógico do PMAQ-AB no âmbito nacional.

A análise dos dados indicou que os documentos oficiais apresentaram o PMAQ$\mathrm{AB}$ em etapas e que muitas definições importantes não foram esclarecidas no início do primeiro ciclo, o que dificultou a compreensão, principalmente, das ações que deveriam ser desenvolvidas pelos profissionais das equipes que aderiram ao programa em 2011. Atualmente o PMAQ-AB encontra-se em seu terceiro ciclo e, apesar de muito recente, algumas modificações têm sido feitas, particularmente para adequar o processo de avaliação externa e garantir uma continuidade entre os ciclos.

Parece evidente que o modelo lógico da teoria do programa é um instrumento importante e pode ser utilizado em diversos estudos na área de avaliação de políticas públicas, servindo como subsídio para a construção de indicadores de desempenho e outros mecanismos de avaliação. Porém, a análise da validade do desenho do programa deve ser realizada como indicação para testar o modelo proposto e identificar possíveis pontos 
de divergências que revelem especificações locais e regionais que podem influenciar a conformação do programa nos distintos municípios brasileiros.

\section{Referências}

ALELUIA, Italo Ricardo Santos; ALMEIDA, Erika Rodrigues; MEDINA, Maria Guadalupe. A coordenação do cuidado pela Atenção Primária à Saúde e o Programa Nacional de Melhoria do Acesso e da Qualidade da Atenção Básica: contribuições para a análise. In: ALMEIDA, Patty Fidelis de; SANTOS, Adriano Maia dos; SOUZA, Mariluce Karla Bomfim de (Org.). Atenção Primária à Saúde na coordenação do cuidado em regiões de saúde. Salvador: EDUFBA, 2015. p. 89-113.

ALSHAMSAN, Riyadh et al. Has pay for performance improved the management of diabetes in the United Kingdom? Primary Care Diabetes, v. 4, p. 73-78, 2010.

BARBOSA, Milena Rocha. Análise do $1^{\circ}$ ciclo do Programa Nacional de Melhoria do Acesso e da Qualidade na Atenção Básica (PMAQ-AB) em um município de médio porte. 2014. 107 f. Dissertação (Mestrado em Política, Gestão e Avaliação do Sistema Único de Saúde) - Faculdade de Medicina, Universidade Federal de Juiz de Fora, Juiz de Fora, Minas Gerais, 2014.

BERENSON, Robert A.; RICH, Eugene. US Approaches to Physician Payment:The Deconstruction of Primary Care. J Gen Intern Med, v. 25, n. 6, p. 613-18, 2010.

BRASIL. Ministério da Saúde. Portaria n. 1.654, de 19 de julho de 2011. Institui, no âmbito do Sistema Único de Saúde, o Programa Nacional de Melhoria do Acesso e da Qualidade da Atenção Básica (PMAQ-AB) e o Incentivo Financeiro do PMAQ-AB, denominado Componente de Qualidade do Piso de Atenção Básica Variável - PABVariável. Diário Oficial da União, Brasília, DF, 20 jul. 2011a. Seção 1, p. 79.

Ministério da Saúde. Portaria n. 576, de 19 de setembro de 2011. Sem título. Diário Oficial da União, Brasília, DF, 20 set. 2011b. Seção 1, p. 23.

Ministério da Saúde. Portaria n. 2.396, de 13 de outubro de 2011. Define o valor mensal integral do incentivo financeiro do (PMAQ-AB), denominado como Componente de Qualidade do Piso de Atenção Básica Variável PABVariável. Diário Oficial da União, Brasília, DF, 14 out. 2011c. Seção 1, p. 49.

Ministério da Saúde. Portaria n. 2.488, de 21 de outubro de 2011. Aprova a Política Nacional de Atenção Básica, estabelecendo a revisão de diretrizes e normas para a organização da Atenção Básica, para a Estratégia Saúde da Família (ESF) e o Programa de Agentes Comunitários de Saúde (PACS). Diário Oficial da União, Brasília, DF, 22 out. 2011 d. Seção 1, p. 58.

. Ministério da Saúde. Portaria n. 2.812, de 29 de novembro de 2011. Homologa a adesão dos Municípios e das respectivas equipes de Atenção Básica ao Programa Nacional de Melhoria do Acesso e da Qualidade da Atenção Básica (PMAQ-AB). Diário Oficial da União, Brasília, DF, 30 nov. 2011e. Seção 1, p. 38.

. Ministério da Saúde. Portaria n. 225, de 10 de fevereiro de 2012. Homologa a adesão dos Municípios e das respectivas Equipes de Atenção Básica ao Programa Nacional de Melhoria do Acesso e da Qualidade da Atenção Básica (PMAQ-AB). Diário Oficial da União, Brasília, DF, 13 fev. 2012 a. Seção 1, p. 85.

Ministério da Saúde. Portaria n. 644, de 10 de abril de 2012. Homologa a adesão dos Municípios e das respectivas Equipes de Atenção Básica ao Programa Nacional de Melhoria do Acesso e da Qualidade da Atenção Básica (PMAQ-AB). Diário Oficial da União, Brasília, DF, 11 abr. 2012b. Seção 1, p.120.

Ministério da Saúde. Portaria n. 866, de 3 de maio de 2012. Altera o prazo para solicitação da avaliação externa no Programa Nacional de Melhoria do Acesso e da Qualidade da Atenção Básica (PMAQ-AB) e as regras de classificação da certificação das equipes participantes do Programa. Diário Oficial da União, Brasília, DF, 4 maio. 2012c. Seção 1, p. 56.

. Ministério da Saúde. Portaria n. 1.089, de 28 de maio de 2012. Define o valor mensal integral do incentivo financeiro do Programa Nacional de Melhoria do Acesso e da Qualidade da Atenção Básica (PMAQ-AB), 
denominado como Componente de Qualidade do Piso de Atenção Básica Variável (PABVariável). Diário Oficial da União, Brasília, DF, 29 maio. 2012d. Seção 1, p. 82.

.Ministério da Saúde.Portaria n.2.626, de 19 de novembro de 2012.Autoriza o repasse do incentivo financeiro do Programa Nacional de Melhoria do Acesso e da Qualidade da Atenção Básica (PMAQ-AB), denominado como Componente de Qualidade do Piso de Atenção Básica Variável. Diário Oficial da União, Brasília, DF, 20 nov. 2012e. Seção 1, p. 28.

Ministério da Saúde. Portaria n.3.114, de 28 de dezembro de 2012.Autoriza o repasse do incentivo financeiro do Programa Nacional de Melhoria do Acesso e da Qualidade da Atenção Básica (PMAQ-AB), denominado como Componente de Qualidade do Piso de Atenção Básica Variável. Diário Oficial da União, Brasília, DF, 31 dez. 2012f. Seção 1, p. 204.

. Ministério da Saúde. Secretaria de Atenção à Saúde. Departamento de Atenção Básica. Programa Nacional de Melhoria do Acesso e da Qualidade da Atenção Básica (PMAQ-AB): manual instrutivo. Brasília: MS, 2012g. 62 p. (Série A. Normas e Manuais Técnicos).

Ministério da Saúde. Secretaria de Atenção à Saúde. Departamento de Atenção Básica. Documento sintese para avaliação externa. Brasília: MS, 2012h. 55 p.

. Ministério da Saúde. Portaria n. 283, de 28 de fevereiro de 2013. Autoriza o repasse do incentivo financeiro do Programa Nacional de Melhoria do Acesso e da Qualidade da Atenção Básica (PMAQ-AB), denominado como Componente de Qualidade do Piso de Atenção Básica Variável. Diário Oficial da União, Brasília, DF, 1 mar. 2013a. Seção 1, p. 51.

. Ministério da Saúde. Portaria n. 535, de 3 de abril de 2013. Altera a Portaria n. 1.654/GM/MS, de 19 de julho de 2011, que institui, no âmbito do Sistema Único de Saúde, o Programa Nacional de Melhoria do Acesso e da Qualidade da Atenção Básica (PMAQ-AB), e a Portaria n. 866/GM/MS, de 3 de maio de 2012, que altera o prazo para solicitação da avaliação externa no Programa Nacional de Melhoria do Acesso e da Qualidade da Atenção Básica (PMAQ-AB). Diário Oficial da União, Brasília, DF, 4 abr. 2013b. Seção 1, p. 35.

Ministério da Saúde. Portaria n. 562, de 4 de abril de 2013. Define o valor mensal integral do incentivo financeiro do Programa Nacional de Melhoria do Acesso e da Qualidade da Atenção Básica (PMAQ-AB-AB), denominado como Componente de Qualidade do Piso de Atenção Básica Variável (PABVariável). Diário Oficial da União, Brasília, DF, 5 abr. 2013c. Seção 1, p. 62.

. Ministério da Saúde. Portaria n. 635, de 17 de abril de 2013. Homologa a adesão dos Municípios ao segundo ciclo do Programa Nacional de Melhoria do Acesso e da Qualidade da Atenção Básica (PMAQ- AB). Diário Oficial da União, Brasília, DF, 18 abr. 2013d. Seção 1, p. 39.

. Ministério da Saúde. Portaria n. 1063, de 3 de junho de 2013. Altera as regras de certificação das equipes participantes do Programa Nacional de Melhoria do Acesso e da Qualidade da Atenção Básica (PMAQ-AB) e do Programa de Melhoria do Acesso e Qualidade dos Centros de Especialidades Odontológicas (PMAQ-CEO). Diário Oficial da União, Brasília, DF, 4 jun. 2013e. Seção 1, p. 49.

.Ministério da Saúde.Portaria n.1383, de 9 de julho de 2013.Homologa a contratualização/ recontratualização dos Municípios ao segundo ciclo do Programa Nacional de Melhoria do Acesso e da Qualidade da Atenção Básica (PMAQ-AB). Diário Oficial da União, Brasília, DF, 10 jul. 2013f. Seção 1, p. 109.

Ministério da Saúde. Secretaria de Atenção à Saúde. Departamento de Atenção Básica. Programa Nacional de Melhoria do Acesso e da Qualidade da Atenção Básica (PMAQ-AB): manual instrutivo. Brasília: MS, 2013g. 38 p.

Ministério da Saúde. Portaria n. 162, de 29 de janeiro de 2014. Altera a Portaria n. 1.383/GM/MS, de 9 de julho de 2013, que homologa a contratualização/recontratualização dos Municípios ao segundo ciclo do Programa Nacional de Melhoria do Acesso e da Qualidade da Atenção Básica (PMAQ-AB). Diário Oficial da União, Brasília, DF, 30 jan. 2014a. Seção 1, p. 79.

Ministério da Saúde. Portaria n. 2.666, de 4 de dezembro de 2014. Autoriza o repasse do incentivo financeiro do Programa Nacional de Melhoria do Acesso e da Qualidade da Atenção Básica (PMAQ-AB), 
denominado como Componente de Qualidade do Piso de Atenção BásicaVariável. Diário Oficial da União, Brasília, DF, 5 dez. 2014b. Seção 1, p. 52.

Ministério da Saúde. Portaria n. 836, de 26 de junho de 2015. Autoriza o repasse do incentivo financeiro do Programa Nacional de Melhoria do Acesso e da Qualidade da Atenção Básica (PMAQ-AB), denominado como Componente de Qualidade do Piso de Atenção Básica Variável, referente à certificação final das equipes participantes do $2^{\circ}$ ciclo do PMAQ-AB. Diário Oficial da União, Brasília, DF, 29 jun. 2015a. Seção 1, p. 52.

Ministério da Saúde. Portaria n. 1.645, de 2 de outubro de 2015. Dispõe sobre o Programa Nacional de Melhoria do Acesso e da Qualidade da Atenção Básica (PMAQ-AB). Diário Oficial da União, Brasília, DF, 5 out. 2015b. Seção 1, p. 668.

Ministério da Saúde. Portaria n. 1.814, de 8 de outubro de 2016. Homologa a contratualização/ recontratualização dos Centros de Especialidades Odontológicas (CEO) ao segundo ciclo do Programa Nacional de Melhoria do Acesso e da Qualidade dos Centros de Especialidades Odontológicas (PMAQ-CEO). Diário Oficial da União, Brasília, DF, 10 out. 2016b. Seção 1, p. 44.

.Ministério da Saúde. Portaria n. 1.658, de 12 de setembro de 2016. Homologa a contratualização/ recontratualização dos Municípios ao terceiro ciclo do Programa Nacional de Melhoria do Acesso e da Qualidade da Atenção Básica (PMAQ-AB). Diário Oficial da União, Brasília, DF, 13 set. 2016a. Seção 1, p. 41.

Ministério da Saúde. Secretaria de Atenção à Saúde. Departamento de Atenção Básica. Programa Nacional de Melhoria do Acesso e da Qualidade da Atenção Básica (PMAQ-AB): manual instrutivo. Brasília: MS, 2017. 89 p.

CAVALCANTI, Pauline Cristine da Silva. Atenção Básica no Brasil: Desafios e Iniciativas para Qualificação. 2014. 133 f. Dissertação (Mestrado em Saúde Coletiva) - Faculdade de Ciências da Saúde, Universidade de Brasília, Brasília, 2014.

CAVALCANTI, Pauline Cristine da Silva; OLIVEIRA NETO, Aristides Vitorino; SOUSA, Maria de Fátima de. Quais são os desafios para a qualificação da Atenção Básica na visão dos gestores municipais? Saúde Debate, Rio de Janeiro, v. 39, n. 105, p. 323-336, 2015.

CHAMPAGNE, François et al. A avaliação no campo da saúde: conceitos e métodos. In: BROUSSELLE, Astrid (Org.). Avaliação: conceitos e métodos. Rio de Janeiro: Editora Fiocruz, 2011.p. 41-60.

COELHO E SILVA, Lais Marques et al. Implementação do Programa de Melhoria do Acesso e Qualidade segundo gestores da Atenção Básica de São Paulo. Acta Paul Enferm., São Paulo, v. 30, n. 4, p. 397-403, 2017.

CRUZ, Marly Marques da et al. Usos do planejamento e autoavaliação nos processos de trabalho das equipes de Saúde da Família na Atenção Básica. Saúde Debate, Rio de Janeiro, v. 38, n. especial, p. 124-139, out. 2014.

FAUSTO, Márcia Cristina Rodrigues et al. A posição da Estratégia Saúde da Família na rede de atenção à saúde na perspectiva das equipes e usuários participantes do PMAQ-AB. Saúde Debate, Rio de Janeiro, v. 38, n. especial, p. 13-33, out. 2014.

FELISBERTO, Eronildo. Da teoria à formulação de uma Política Nacional de Avaliação em Saúde: reabrindo o debate. Ciência \& Saúde Coletiva, Rio de Janeiro, v. 1, n. 3, p. 553-563, 2006.

GIRARDI, Sábado Nicolau; SALGADO, Valéria Alpino Bigonha; VAN STRALEN, Ana Cristina de Sousa. Aspectos críticos da experiência municipal com remuneração variável de equipes de Atenção Básica em saúde. Congresso de Gestão Pública - CONSAD,VII., 2015, Brasília.

GOMES, Wanessa da Silva. Programa de melhoria do acesso e qualidade da atenção básica: reflexões sobre o papel da avaliação na efetivação da integralidade em saúde. 2013. 63 f. Dissertação (Mestrado em Saúde Coletiva) Programa de Pós-graduação Integrado em Saúde Coletiva, Universidade Federal de Pernambuco, Recife, 2013.

KONDO, Karli et al. Implementation Processes and Pay for Performance in Healthcare: A Systematic Review. $J$ Gen Intern Med., v. 31, supl. 1, p. 61-9, 2016.

LINHARES, Paulo Henrique Arruda; LIRA, Geison Vasconelos; ALBUQUERQUE, Izabelle Mont'Alverne Napoleão. Avaliação do Programa Nacional de Melhoria do Acesso e da Qualidade da Atenção Básica no estado do Ceará. Saúde debate, Rio de Janeiro, v. 38, n. especial, p. 195-208, out. 2014. 
LOPES, Estela Auxiliadora Almeida. O Programa Nacional de Melhoria do Acesso e da Qualidade na Atenção Básica e seu potencial de gerar mudanças no trabalho dos profissionais. 2013. 92 f.Dissertação (Mestrado em Saúde Coletiva) - Faculdade de Ciências da Saúde, Universidade de Brasília, Brasília, 2013.

LORENA SOBRINHO,José Eudes de et al.Acesso e qualidade:avaliação das Equipes de Saúde Bucal participantes do PMAQ-AB 2012 em Pernambuco. Saúde em Debate, Rio de Janeiro, v. 39, n. 104, p. 136-146, jan./mar. 2015.

MACHADO, Jacinta de Fátima Franco Pereira et al. Educação Permanente no cotidiano da Atenção Básica no Mato Grosso do Sul. Saúde Debate, Rio de Janeiro, v. 39, n. 104, p. 102-113, jan./mar. 2015.

MACINKO, James; HARRIS, Matthew J.; ROCHA, Maria Gomes. Brazil's National Program for Improving Primary Care Access and Quality (PMAQ-AB): Fulfilling the Potential of the World's Largest Payment for Performance System in Primary Care.J Ambulatory Care Manage, v. 40, n. 2 Supp, p. S4-S11, maio./jun. 2017.

MORAES, Paulo Navarro; IGUTI,Aparecida Mari.Avaliação do desempenho do trabalhador como forma peculiar de prescrição do trabalho: uma análise do PMAQ-AB. Saúde em Debate, Rio de Janeiro, v. 37, n. 98, p. 416-426, jul./set. 2013.

MOTA, Roberta Rodrigues de Alencar;DAVID, Helena Maria Scherlowski Leal. Programa Nacional de Melhoria do Acesso e da Qualidade da Atenção Básica: questões a problematizar. Rev. Enferm. UERJ, Rio de Janeiro, v. 23, n. 1, p. 122-7, jan./fev. 2015.

NEVES, Teresa Cristina de Carvalho Lima; MONTENEGRO, Luiz Albérico Araújo; BITTENCOURT, Sonia Duarte de Azevedo. Produção e registro de informações em saúde no Brasil: panorama descritivo através do PMAQ-AB. Saúde Debate, Rio de Janeiro, v. 38, n. 103, p. 756-770, out./dez. 2014.

PEARSON, Steven D. et al.The Impact of Pay-for-Performance on Health Care Quality in Massachusetts, 20012003. Health Affairs, v. 27, n. 4, p. 1167-76, 2008.

PERELMAN, Julian et al. Pagamento pelo Desempenho nos Cuidados de Saúde Primários. Coimbra: Editora Almedina, 2016.

PINTO, Hêider Aurélio. Múltiplos olhares sobre e a partir do Programa Nacional de Melhoria do Acesso e da Qualidade na Atenção Básica. 2014. 231 f. Dissertação (Mestrado em Saúde Coletiva) - Escola de Enfermagem, Universidade Federal do Rio Grande do Sul, Porto Alegre, 2014.

PINTO, Hêider Aurélio; SOUSA, Allan Nuno Alves de; FERLA, Alcindo Antonio. O Programa Nacional de Melhoria do Acesso e da Qualidade da Atenção Básica: várias faces de uma política inovadora. Saúde Debate, Rio de Janeiro, v. 38, n. especial, p. 358-372, out. 2014.

PINTO, Hêider Aurélio; SOUSA, Allan Nuno Alves de; FLORÊNCIO, Alexandre Ramos. O Programa Nacional de Melhoria do Acesso e da Qualidade da Atenção Básica: reflexões sobre o seu desenho e processo de implantação. RECIIS: Revista eletrônica de comunicação, informação e inovação em saúde, Rio de Janeiro, v. 6, n. 2, supl., ago. 2012.

ROCHA, Erika Maria Sampaio et al. Programa de Melhoria do Acesso e da Qualidade da Atenção Básica: ambiguidades no discurso oficial. Rev. Bras. Pesq. Saúde,Vitória, v. 18, n. 1, p. 21-35, jan./mar. 2016.

RODRIGUES, Valéria; SANTOS, Cleber Ronald Inácio dos; PEREIRA, Mariana Uchoa. A experiência de planejar e operacionalizar o PMAQ-AB no estado do Acre. Saúde debate, Rio de Janeiro, v. 38, n. especial, p. 173181, out. 2014.

ROSSI, Peter; LIPSEY, Mark; FREEMAN, Howard. Evaluation, a Systematic Approach. Thousand Oaks, EUA: Sage Publications, 2004.

SANTOS, Adilson Ribeiro dos et al. Programa de Melhoria do Acesso e da Qualidade da Atenção Básica: em busca de novos sentidos no processo de trabalho. Rev.Saúde.Com., v. 10, n. 3, p. 307-314, 2014.

SOUSA, Elisabete Maria de Jesus Teixeira de. Pagamento em Função do Desempenho nos Cuidados de Saúde Primários: Três Serviços Nacionais de Saúde, Três Experiências. 2011. 110 f. Dissertação (Mestrado em Saúde Pública) Escola Nacional de Saúde Pública, Universidade Nova de Lisboa, Lisboa, 2011. 
SOUZA, Marize Barros de et al.Trabalho em equipe na atenção primária: a experiência de Portugal. Rev. Panam. Salud Publica, v. 33, n. 3, p. 190-195, 2013.

TEIXEIRA, Mirna Barros et al. Avaliação das práticas de promoção da saúde: um olhar das equipes participantes do Programa Nacional de Melhoria do Acesso e da Qualidade da Atenção Básica. Saúde debate, Rio de Janeiro, v. 38, p. 52-68, out. 2014.

TOMASI, Elaine et al. Estrutura e processo de trabalho na prevenção do câncer de colo de útero na Atenção Básica à Saúde no Brasil: Programa de Melhoria do Acesso e da Qualidade - PMAQ-AB. Rev. Bras. Saúde Matern. Infant., Recife, v. 15, n. 2, p. 171-180, abr./jun. 2015. 


\title{
National Program for the Improvement of Access and Quality of Primary Care (PMAQ-AB): Policy Modeling at the National Level
}

\begin{abstract}
This article aimed to identify the constituent elements of the theoretical framework of PMAQ$\mathrm{AB}$ and, through this, to systematize the logical model of this program. For that, an evaluative study was carried out that contemplated a review and documentary analysis that included 35 documents related to intervention at the national level. From the elaboration of the model it was possible to understand the logical plausibility of the proposal. It can be presumed that this result will allow the extension of the evaluative vision to the organizational issues related to the program, making possible a comparison between the program as foreseen and what has in fact been implemented in the different contexts of the Brazilian municipalities.
\end{abstract}

Keywords: Primary health care, health policy, health assessment, performance evaluation, evaluation of the quality of health care.

\section{Programa Nacional de Mejora del Acceso y de la Calidad de la Atención Básica (PMAQ-AB): modelización de la política a nivel nacional}

\section{Resumen}

Este artículo objetivó identificar los elementos constituyentes del marco teórico del PMAQ-AB y, a través de ello, sistematizar el modelo lógico de este programa. Para ello, se realizó un estudio evaluativo que contempló una revisión y análisis documental que incluyó 35 documentos relativos a la intervención propuesta a nivel nacional. A partir de la elaboración del modelo fue posible comprender la plausibilidad lógica de la propuesta. Se puede presumir que ese resultado permitirá la ampliación de la visión de evaluación para las cuestiones organizacionales relacionadas con el programa, al viabilizar una comparación entre el programa como se había previsto y lo que ha sido, de hecho, implantado en los diversos contextos de los municipios brasileños.

Palabras clave: Atención primaria a la salud, política de salud, evaluación en salud, evaluación del desempeño, evaluación de la calidad del cuidado de la salud. 